FOLIA

Amazónica

Revista del Instituto de Investigaciones

de la Amazonía Peruana

\title{
SILVICULTURA DE UN RODAL DE REGENERACIÓN NATURAL DE Calycophyllum spruceanum (Benth.) Hook. f. ex K. Schum (RUBIACEAE) EN LA LLANURA ALUVIAL INUNDABLE DE IQUITOS, AMAZONÍA PERUANA
}

\author{
Luis FREITAS ALVARAD0 ${ }^{1, *}$, Ricardo ZÁRATE GÓMEZ1, Dennis DEL \\ CASTILLO TORRES ${ }^{1}$, Ander DÁVILA DÍAZ ${ }^{1}$, Carlos VILLACORTA GONZÁLES ${ }^{2}$, \\ Julinho BENAVIDES RÍOS ${ }^{1}$
}

\footnotetext{
1 Instituto de Investigaciones de la Amazonía Peruana - IIAP. Iquitos, Perú.

2 CITE Forestal, Instituto Tecnológico de la Producción - ITP. Iquitos, Perú.

* Correo electrónico: Ifreitas@iiap.gob.pe
}

\section{RESUMEN}

Los rodales de Calycophyllum spruceanum (Benth.) Hook. f. ex K. Schum., conocidos localmente como capironales, ocurren naturalmente en la planicie de inundación de los ríos principales de la Amazonía baja peruana. En el presente estudio, se caracterizó la estructura horizontal y se analizó la condición silvicultural de un rodal de capirona ubicado en el distrito de Belén, departamento de Loreto. Se realizó un inventario forestal y se midieron los parámetros sobre la condición silvicultural de las plantas según categorías de tamaño. El rodal tiene 8 años y presenta 961 individuos, 7,68 $\mathrm{m}^{2}$ de área basal y un volumen de $25,68 \mathrm{~m}^{3}$. La condición silvicultural según iluminación de copas muestra que el $40,48 \%$ de la población se encuentra en condiciones deficientes de luz y pertenecen al grupo de latizales, esta situación también afecta la forma de las copas, ya que dentro de este grupo el 33,51\% corresponden a la categoría tolerable con medio círculo de copa y un 22,27 son copas pobres a muy pobres. En cuanto a la calidad de fuste el $30,07 \%$ de individuos presentan fustes bien rectos y cilíndricos y $25,7 \%$ con fuste recto en la mayor parte de su longitud. El análisis silvicultural del rodal muestra que existe un alto número de plantas con deficiente iluminación y forma de copas, por lo que se considera que el 
tratamiento silvicultural adecuado es un raleo de tipo selectivo-elegido con fin de regular el distanciamiento entre las plantas mejor conformadas y vigorosas y mejorar las condiciones para su desarrollo.

PALABRAS CLAVE: Manejo forestal, producción maderable, estructura de la vegetación.

\title{
SILVICULTURE OF NATURAL REGENERATION STAND OF Calycophyllum spruceanum (Benth.) Hook. f. ex K. Schum (RUBIACEAE) IN ALLUVIAL FLOODPLAIN OF IQUITOS, PERUVIAN AMAZON
}

\begin{abstract}
Horizontal structure and silvicultural conditions of a naturally-regenerated Calycophyllum spruceanum (known in Peru as "Capirona") stand in an alluvial floodplain were assessed. A forestry inventory was carried out and silvicultural conditions parameters were measured according their size categories. C. spruceanum stand has 961 individuals, $7.68 \mathrm{~m} 2$ of basal area and $25.68 \mathrm{~m} 3$ of volume. Silvicultural condition by crown lighting indicated that $40.48 \%$ of total population has deficient lighting and belongs to the latizales class. This deficient lighting also affected the crown shape because $33.51 \%$ of the stand belong to the "tolerable" class with half-crown circle and $22.27 \%$ varies from poor to very poor crown. Regarding the stem quality, $30.07 \%$ of individuals shows well straight and cylindrical stems and $25.7 \%$ with straight stems in higher of its length. The silvicultural analysis of the stand shows that there are a high number of plants with poor lighting and crown shape, so it is considered that the appropriate silvicultural treatment is a thinning of the selective-chosen type in order to regulate the distance between the best shaped plants and vigorous and improve the conditions for their development.
\end{abstract}

KEYWORDS: Forest management, timber production, vegetation structure. 


\section{INTRODUCCIÓN}

Los bosques de la llanura aluvial inundable en la Amazonía baja peruana están ubicados en las márgenes de los grandes ríos y tributarios, cubriendo aproximadamente 16172884 hectáreas (MINAM, 2015). La actividad forestal maderera en la región Loreto se realiza principalmente en estos bosques y de donde se extrae tradicionalmente las especies maderables como Swietenia macrophylla (caoba), Cedrela odorata (cedro), Ceiba pentandra (lupuna), Hura crepitans (catahua), Calophyllum brasiliense (Lagarto Caspi), siendo afectadas algunas de estas especies debido a la sobreexplotación (Kvist \& Nebel, 2001).

La conservación de los bosques inundables representa un gran reto para los gobiernos regionales quienes necesitan implementar estrategias adecuadas para su manejo y frenar la sobreexplotación de las principales especies maderables. En esta perspectiva el manejo de Calycophyllum spruceanum (Capirona), especie típica de los bosques de la llanura aluvial inundable tiene muchas ventajas y en particular porque aparecen de manera natural, son dominados por una sola especie, con individuos de forma ideal para la construcción, y el uso de leña de manera local y/o comercial. Así mismo, presenta una capacidad de rebrote del tronco cortado y un crecimiento rápido en espacios perturbados naturales por eventos y procesos fluviales y por las prácticas de agricultura en una pequeña escala (Sears, 2003).

Los agricultores silvicultores de la Amazonía peruana pueden contribuir al sector forestal a través de la producción sustentable de esta destacada especie maderable (Orrego \& Bustamante, 2017; Cornelius etal., 2018; Correa et al. 2019). Una de las características de la capirona es tolerar los periodos largos de inundación (3-4 meses), por tal motivo juega un rol importante en la adaptación al cambio climático. Se caracteriza por tener una gran capacidad de almacenamiento y secuestro de carbono. Por ejemplo, un rodal natural de capirona puede almacenar 125,9 t/ha (Gorbitz, 2015).

En los últimos años, la capirona ha presentado una demanda creciente en el mercado internacional similar a la demanda por la madera dura para pisos. Anivel nacional, tiene un alto valor comercial similar a las especies de shihuahuaco (Dipteryx sp.) y estoraque (Myroxylon toleiferum). Sin embargo, la capirona tiene el potencial de ofertar un mayor volumen maderable en trozas. Asimismo, la madera de capirona es dura y se usa para pisos, parquet, molduras, carrocerías, tornería, muebles, molduras, revestimiento, ebanistería, artesanía, carpintería de obras y de interiores (Ugarte \& Domínguez, 2010).

El programa de domesticación del Centro Internacional para la Investigación en Agroforestería en América Latina (ICRAF) desarrolló un programa de domesticación de árboles, habiéndose considerado a $C$. spruceanum como una especie prioritaria para la agroforestería en la Amazonía peruana (Weber, 1999). Sin embargo, se conoce poco de las experiencias prácticas sobre el establecimiento y manejo de capirona en áreas inundables desarrollados por los pobladores ribereños. Por lo tanto, estas experiencias merecen ser estudiadas, registradas y documentadas para obtener información básica social y técnica con el fin de incorporar a la especie en programas de conservación y aprovechamiento sostenible (Correa et al., 2019). Por lo tanto, en el presente estudio presentamos los resultados de la evaluación de un rodal natural de capirona manejado por el Sr. Brígido Rengifo Terán en el distrito de Belén, departamento de Loreto. Para esto, caracterizamos la estructura horizontal haciendo un inventario al $100 \%$ de los individuos y analizamos la condición silvicultural a partir de la iluminación de las copas, forma de 
las copas y calidad de fuste del rodal. Finalmente, damos recomendaciones sobre el manejo y actividades silviculturales para incrementar el crecimiento de los fustes en el rodal.

\section{MATERIAL Y MÉTODOS}

\section{ZONA DE ESTUDIO}

El estudio se realizó en el fundo "Cuerito", en la comunidad de "Moena Caño", Distrito de Belén, Provincia de Maynas, Departamento de Loreto,

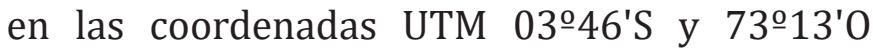
(Figura 1). La fisiografía de la zona corresponde a una planicie de inundación, donde se observan diversas unidades características de la región, tales como islas, meandros, complejo de orillales y terrazas bajas con diferentes tipos de drenaje (MINAM, 2015). Los suelos de estas unidades fisiográficas están influenciados por la dinámica del río y según su capacidad de uso mayor son aptos para cultivo en limpio, cultivo permanente, uso forestal y de protección (Rodríguez et al., 1991).

Climáticamente, la región de Iquitos se caracteriza como cálida y húmeda, con precipitación promedio anual de $3087 \mathrm{~mm}$, distribuidos de tal forma de que no hay una estación seca definida. En lo que se refiere al régimen térmico, la temperatura promedio

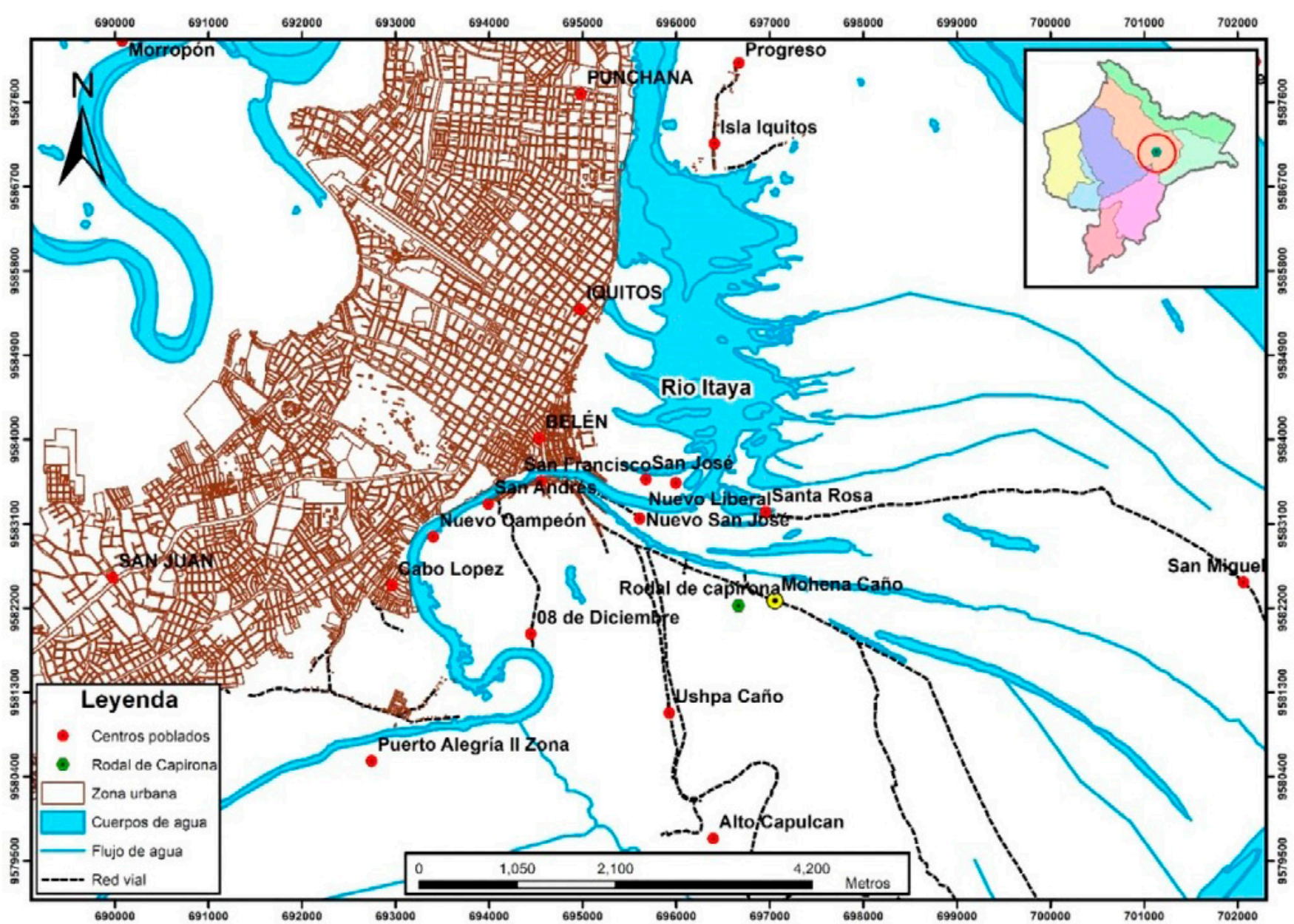

Figura 1: Mapa de ubicación de "Moena Caño", Distrito de Belén, Provincia de Maynas, Departamento de Loreto. 
excede a los $24{ }^{\circ} \mathrm{C}$, con temperatura máxima entre $28-30{ }^{\circ} \mathrm{C}$ y mínima entre $17-20{ }^{\circ} \mathrm{C}$ (Marengo, 1998, para la ciudad de Iquitos, ubicada aproximadamente a un km del área de estudio); con los siguientes microclimas: Clima moderadamente a ligeramente húmedo, cálido a extremadamente cálido (Tello et al., 2013).

\section{DESCRIPCIÓN DE LA FINCA “CUERITO”, HISTORIA DEL RODAL}

La finca donde se ubica el rodal pertenece al Sr. Brígido Rengifo Terán, quién junto a su familia se dedican a la agricultura de subsistencia. La propiedad por estar localizada en zonas inundables, aún no cuenta con título de propiedad, pero está en gestión por más de 30 años. La superficie del predio es de 15 ha, y se inunda anualmente por aguas del río Amazonas entre los meses de enero a mayo. En la época de estiaje del año 2009, se instaló una pequeña chacra de aproximadamente 1 ha, donde sembró productos agrícolas anuales, a finales del año después de cosechar los productos, nuevamente se limpió y habilitó el terreno para la campaña 2010. Al retomar a la chacra habilitada, se encontró que el terreno estaba totalmente cubierto por plántulas de regeneración natural de capirona y se decidió no instalar más cultivos. El rodal ha seguido su proceso natural de sucesión y ha sido sometido a limpiezas periódicas, desde los cuatro años de edad la madera viene siendo aprovechada de acuerdo a las necesidades y requerimientos de terceros y vendiendo la madera rolliza para construcciones rurales (Figura 2).

\section{DISEÑO DE LA INVESTIGACIÓN}

Para facilitar el levantamiento de la vegetación se delimitó el área del rodal uniendo la ubicación de las plantas de los bordes, dando como resultado la forma de un polígono la cual fue "encerrada" en un rectángulo en el terreno (Figura 3). Para el inventario de la vegetación se utilizó el método

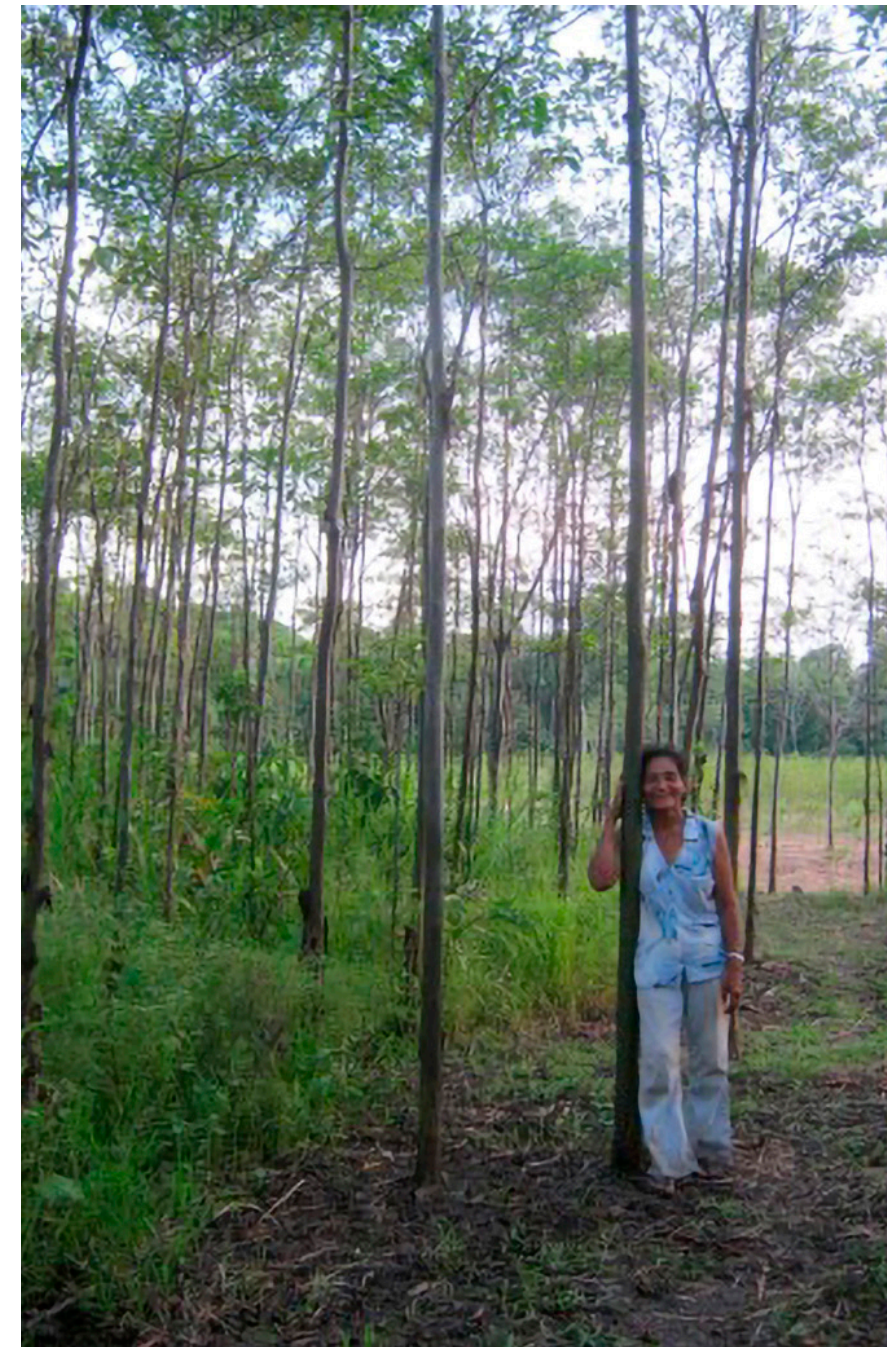

Figura 2: Rodal de capirona Calycophyllum spruceanum en Moena Caño, distrito de Belén, Amazonía peruana.

de muestreo sistemático irrestricto mediante fajas longitudinales de $10 \mathrm{~m}$ de ancho y diferente longitud, ubicadas a lo largo de uno de los lados del rectángulo (Figura 3).

En diciembre del año 2018, se realizó un inventario forestal al $100 \%$ de todas las plantas de capirona, diferenciando los individuos según tres estadios de desarrollo o tamaños de la planta: 1) brinzales, plantas de 0,3-1,4 m de altura; 2) latizales, plantas de $\geq 1,5 \mathrm{~m}$ de altura $\mathrm{y}<10 \mathrm{~cm}$ de DAP (diámetro a la altura del pecho); 3 ) fustales, plantas de $\geq 10 \mathrm{~cm}$ de DAP. 


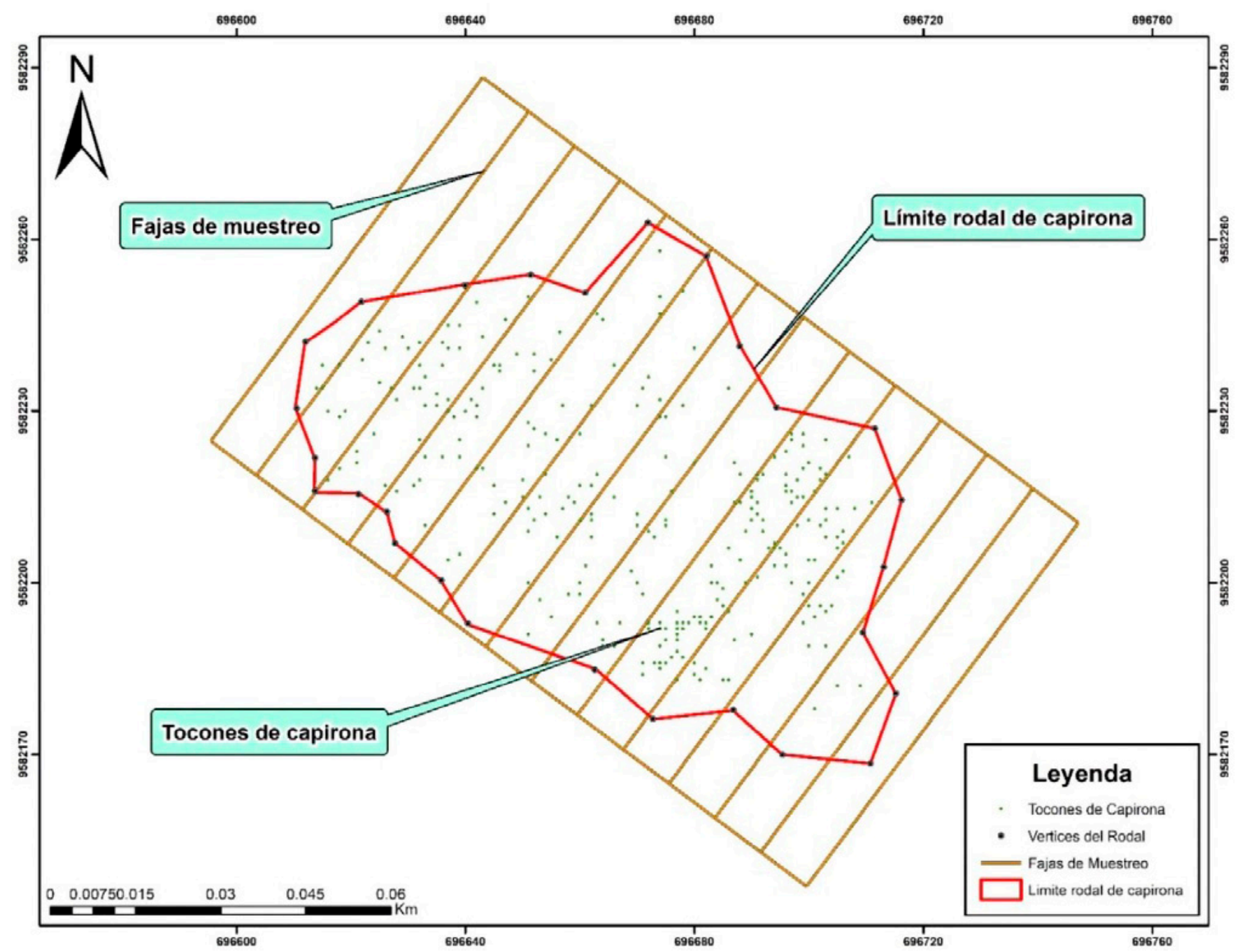

Figura 3: Diseño de la parcela de muestreo.

\section{LEVANTAMIENTO DE LA VEGETACIÓN}

El levantamiento de la vegetación se desarrolló de acuerdo a la metodología planteada por Synnott (1991) y utilizando los siguientes parámetros para los brinzales: número de planta, altura (m), iluminación de la copa; y para los latizales y fustales: número de planta, diámetro a la altura del pecho (DAP $\mathrm{cm}$ ), altura total $(\mathrm{m})$, altura comercial $(\mathrm{m})$, diámetro de copa $(\mathrm{m})$, iluminación de la copa, forma de la copa y calidad de fuste.

La iluminación de copa estima la cantidad de luz que recibe un árbol, y fue evaluada de la siguiente forma: (1) Luz plena vertical y lateral (Buena), (2) Luz plena vertical (Buena), (3) Alguna luz vertical (Aceptable), (4) Luz lateral (Deficiente) y (5) Sin luz vertical ni lateral (Deficiente).

La forma de la copa da una indicación del desarrollo y posible vitalidad actual del árbol en relación con su medio ambiente. Se evaluó de la siguiente forma: (1) Círculo completo (Perfecta), (2) Círculo irregular (Buena), (3) Medio círculo (Tolerable), (4) Menos de medio círculo (Pobre) y (5) Solo una o pocas ramas (Muy pobre). 
Calidad del fuste define tentativamente el valor comercial potencial del árbol, y se evaluó de la siguiente forma: (1) Fuste completamente recto y circular en las secciones, cilíndrico, sin defectos; (2) Fuste bien recto y cilíndrico, bastante circular en secciones, sin defectos; (3) Fuste recto en la mayor parte de su longitud, ligeramente cónico y parcialmente circular, sin defectos, buena madera aserrada; (4). Fuste regular, más o menos recto en algunos metros más o menos circular, sin defectos importantes, parcialmente utilizable para madera aserrada; (5) Fuste poco regular, de crecimiento ligeramente espiralado, algo torcido, bifurcado, sin defectos importantes (6) Fuste de crecimiento espiralado, torcido, muy bifurcado, achaparrado.

Debidoa que elrodalviene siendo aprovechado, se realizó también el conteo y ubicación de los tocones (parte de la base del árbol que queda después del aprovechamiento) utilizando un GPS y coordenadas UTM.

\section{PROCESAMIENTO Y ANÁLISIS DE LA INFORMACIÓN}

Caracterización estructural: Se analizaron los rasgos de la estructura horizontal expresada en términos de la abundancia, área basal y volumen por clases diamétricas de los individuos del rodal. La abundancia (N) se determinó como la suma de todos los individuos inventariados en el rodal. El área basal (g) de un árbol fue calculado mediante la siguiente fórmula: $g=(\pi / 4) \times \mathrm{d}^{2}$. Dónde: $g=$ área basal de un árbol, $\mathrm{d}=$ diámetro a la altura del pecho. La suma de las áreas basales de los individuos constituye el área basal total del rodal.

El volumen (V) individual de un árbol fue calculado mediante la siguiente fórmula: $\mathrm{V}=$ 0,7854 x $\mathrm{DAP}^{2}$ x Hc x f. Dónde: DAP= diámetro a la altura del pecho, $\mathrm{Hc}=$ altura comercial, $\mathrm{f}=$ factor de forma (igual a 0,7 ). La suma de los volúmenes individuales constituye el volumen total del rodal. Se presentan los valores del número de árboles/ ha, área basal/ha y volumen/ha, calculados a partir de la extrapolación de los datos tomando como referencia el área de muestreo de 0,64 ha.

Condición Silvicultural: La condición silvicultural de todos los árboles fue analizada de acuerdo a distribuciones diamétricas con intervalos de clases de $5 \mathrm{~cm}$, y según categorías de vegetación (brinzales, latizales y fustales) relacionados con la iluminación de copas, forma de copa y calidad de fuste. Así mismo se analizó la distribución del número de árboles, área basal y volumen por clases diamétricas según intervalos de clase de $5 \mathrm{~cm}$.

\section{RESULTADOS}

\section{ESTRUCTURA HORIZONTAL DEL RODAL DE}

Calycophyllum spruceanum

Número de árboles por clases diamétricas: $\mathrm{El}$ número total de individuos en todo el rodal es igual a 961 y 1501/ha, la distribución muestra aproximadamente la forma de una campana, con el mayor número de individuos en la categoría diamétrica de 5-9,9 cm. Al excluir los individuos con menos de $5 \mathrm{~cm}$ de dap, la distribución muestra una curva en forma de "J" invertida, con una mayor concentración del número de árboles también en la clase diamétrica de 5 a 9,9 cm, con 521 individuos (Figura 4). Son escasos los individuos con dap mayor que $20 \mathrm{~cm}$ y suman 36 , mientras existen solamente 3 individuos con DAP mayor a $30 \mathrm{~cm}$ siendo el individuo de mayor diámetro de $32,98 \mathrm{~cm}$.

Área basal $\left(\mathrm{m}^{2}\right)$ por clases diamétricas: El área basal total del rodal es de 7,68 $\mathrm{m}^{2}$, aproximadamente $12 \mathrm{~m}^{2} / \mathrm{ha}$, la distribución muestra una reducción continua del área basal a medida que disminuyen los diámetros, igual a la distribución del número de árboles. Una parte significativa del área basal total se concentra en el grupo de latizales que comprende al mayor 


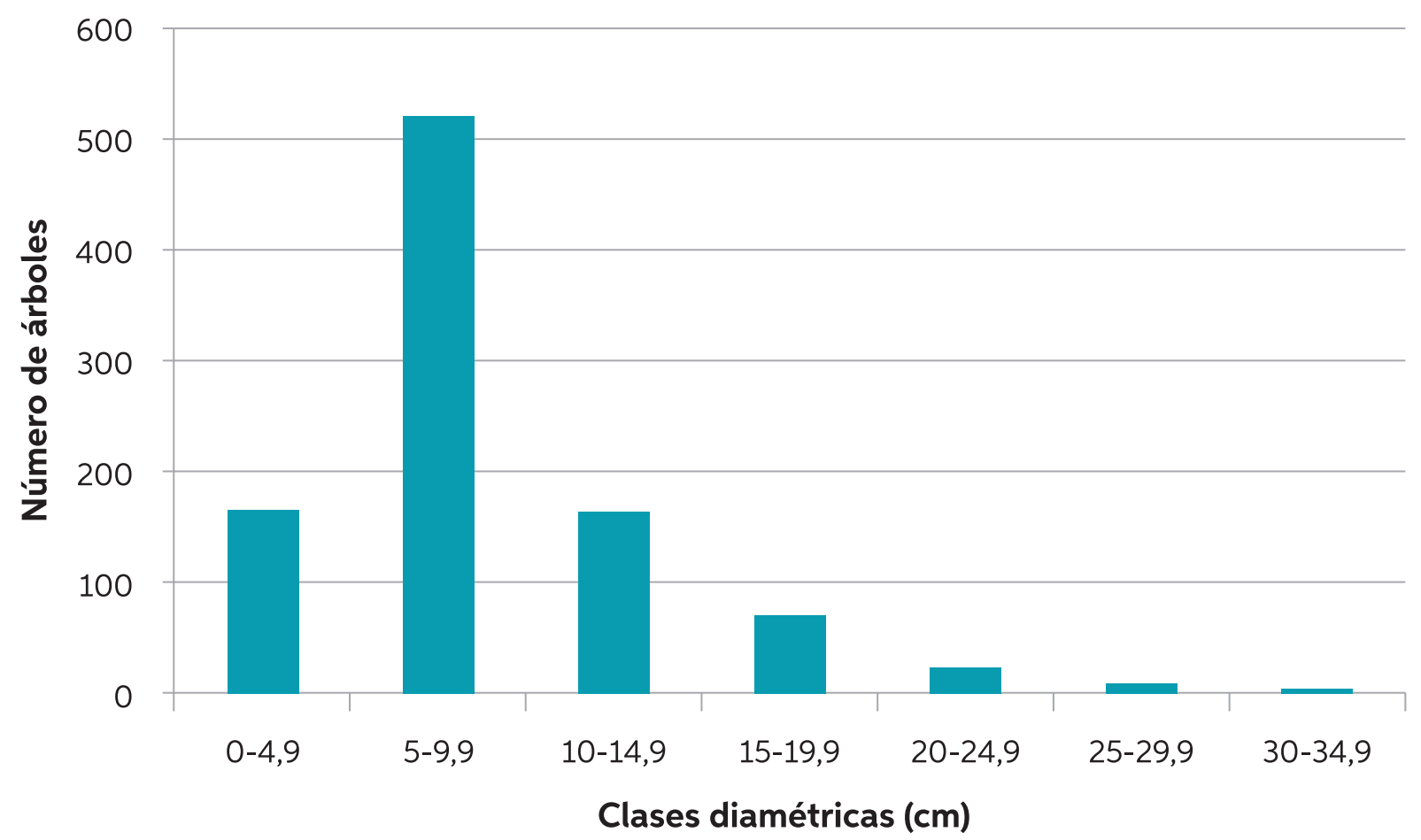

Figura 4: Distribución del número de árboles de Calycophyllum spruceanum por clases diamétricas en el bosque inundable en Belén, Amazonía peruana ( $\mathrm{n}=961)$.

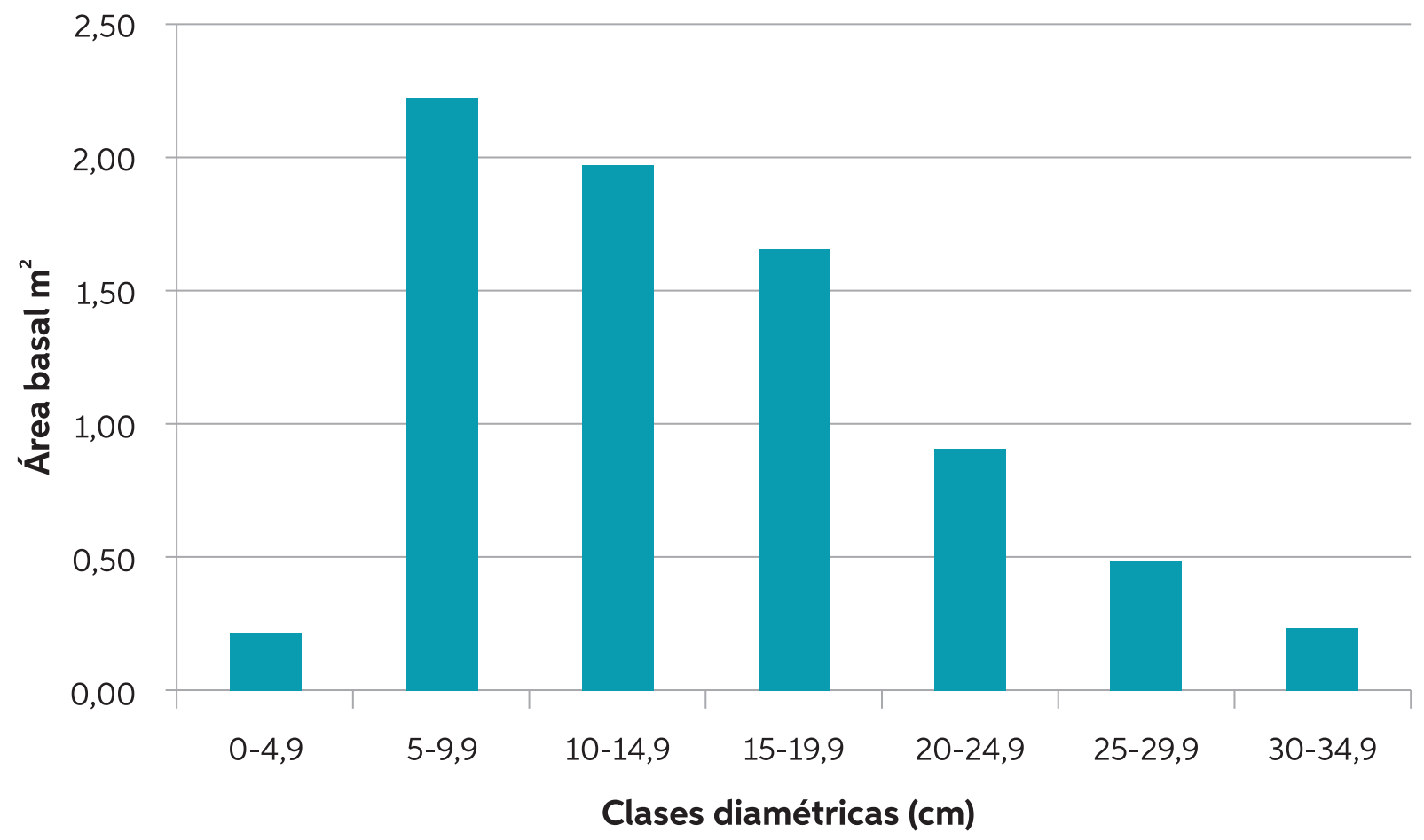

Figura 5: Distribución del área basal por clases diamétricas de Calycophyllum spruceanum en el bosque inundable en Belén, Amazonía peruana $(n=961)$. 


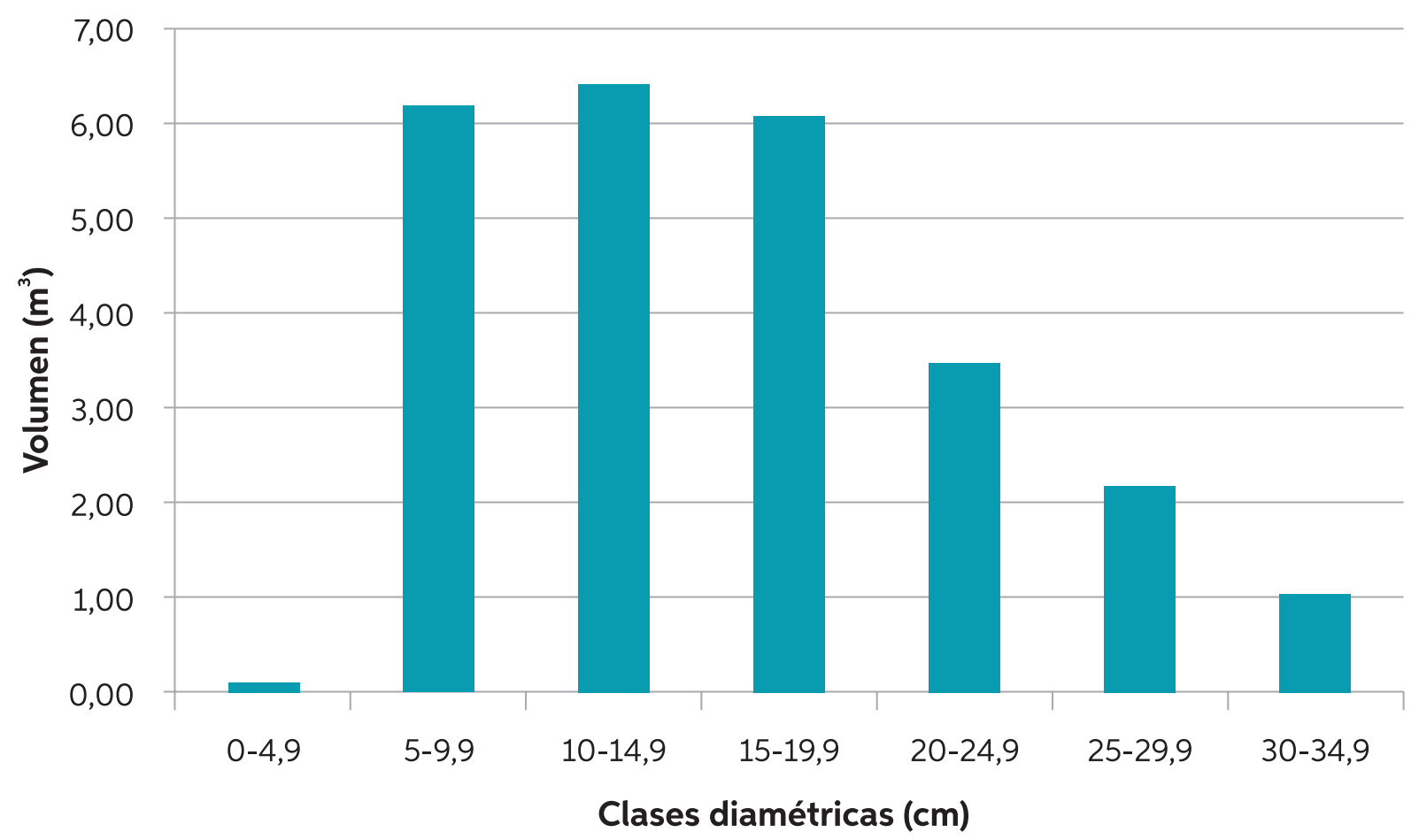

Figura 6: Distribución del volumen maderable por clases diamétricas de Calycophyllum spruceanum en el bosque inundable en Belén, Amazonía peruana $(n=961)$.

número de árboles del rodal, aportando el $28,78 \%$ del área basal total. Los fustales que comprende individuos mayores a $10 \mathrm{~cm}$ de DAP son los que aportan mayor cantidad de área basal con 68,36\%, principalmente por presentar árboles de mayor diámetro (Figura 5).

Volumen $\left(\mathrm{m}^{3}\right)$ por clases diamétricas: $\mathrm{El}$ volumen total que presenta el rodal es de 25,68 $\mathrm{m}^{3}\left(40,13 \mathrm{~m}^{3} / \mathrm{ha}\right)$. La distribución por clases diamétricas es similar a lo que ocurre con el área basal; los latizales aportan el $24,26 \%$ del volumen total, mientras que los fustales aportan el $75,23 \%$. En forma general las clases diamétricas que definen la estructura volumétrica del rodal comprende los árboles entre 5 y $20 \mathrm{~cm}$ de dap que aportan el 73,13\% del volumen total, mientras que el aporte de los individuos menores de $5 \mathrm{~cm}$ de dap es prácticamente insignificante, 0,51\% (Figura 6).
CONDICIÓN SILVICULTURAL DEL RODAL DE Calycophyllum spruceanum

El análisis de la iluminación de copas relacionado con la población total del rodal muestra que la mayor cantidad de individuos pertenecientes a la categoría fustales presentan buena iluminación de copas, el 16,75\% y 7,39\% están recibiendo luz plena vertical y lateral y luz plena vertical respectivamente. En el caso de los latizales que representan el mayor número de árboles $(71 \%)$, la situación es contraria, solamente el 12,69\% presenta luz plena vertical mientras que la mayor cantidad de copas se encuentran en condiciones deficientes, así, el 13,84\% y 26,64\% presentan clases de iluminación con alguna luz vertical y sin luz vertical ni lateral, respectivamente (Tabla 1 ).

En cuanto a las formas de las copas, la mayor cantidad de individuos de la categoría fustales se encuentran en las categorías buena y tolerable, 
Tabla 1: Condición silvicultural según categorías de vegetación e iluminación de copa. Dónde: Clase de iluminación: (1) Luz plena vertical y lateral (Buena), (2) Luz plena vertical (Buena), (3) Alguna luz vertical (Aceptable), (4) Luz lateral (Deficiente) y (5) Sin luz vertical ni lateral (Deficiente).

\begin{tabular}{lccccccc}
\hline \multirow{2}{*}{ Categoría } & \multicolumn{4}{c}{ Clase de iluminación } & \multicolumn{2}{c}{$N^{\circ}$ individuos } \\
\cline { 2 - 8 } & 1 & 2 & 3 & 4 & 5 & Total & $\%$ \\
\hline Fustales & 161 & 71 & 30 & 9 & 2 & 273 & 28,4 \\
Latizales & 95 & 122 & 133 & 76 & 256 & 682 & 71,0 \\
Brinzales & 1 & 0 & 0 & 4 & 1 & 6 & 0,6 \\
Total & 257 & 193 & 163 & 89 & 259 & 961 & 100,0 \\
\hline$\%$ & 26,7 & 20,1 & 17,0 & 9,3 & 26,9 & 100,0 & 100,0 \\
\hline
\end{tabular}

Tabla 2: Condición silvicultural según forma de la copa y categorías de vegetación. Dónde: (1) Círculo completo (Perfecta), (2) Círculo irregular (Buena), (3) Medio círculo (Tolerable), (4) Menos de medio círculo Pobre) y (5) Solo una o pocas ramas (Muy pobre).

\begin{tabular}{lccccccc}
\hline \multirow{2}{*}{ Categoría } & \multicolumn{4}{c}{ Clase según forma de copa } & \multicolumn{2}{c}{$N^{\circ}$ individuos } \\
\cline { 2 - 8 } & 1 & 2 & 3 & 4 & 5 & Total & $\%$ \\
\hline Fustales & 4 & 100 & 119 & 49 & 1 & 273 & 28,4 \\
Latizales & 1 & 145 & 322 & 164 & 50 & 682 & 71,0 \\
Brinzales & 0 & 1 & 5 & 0 & 0 & 6 & 0,6 \\
Total & 5 & 246 & 446 & 213 & 51 & 961 & 100,0 \\
\hline$\%$ & 0,5 & 25,6 & 46,4 & 22,2 & 5,3 & 100,0 & 100,0 \\
\hline
\end{tabular}

Tabla 3: Condición silvicultural según calidad de fuste y categorías de vegetación. Dónde: (1) Fuste completamente recto y circular, cilíndrico, sin defectos; (2) Fuste bien recto y cilíndrico, sin defectos;

(3) Fuste recto en la mayor parte de su longitud, ligeramente cónico y parcialmente circular, sin defectos, buena madera aserrada; (4) Fuste regular, más o menos recto más o menos circular en algunos metros, sin defectos importantes, parcialmente para madera aserrada; (5) Fuste poco regular, de crecimiento ligeramente espiralado, algo torcido, bifurcado, sin defectos importantes; (6) Fuste de crecimiento espiralado, torcido, muy bifurcado, achaparrado.

\begin{tabular}{lcccccccc}
\hline \multirow{2}{*}{ Categoría } & \multicolumn{9}{c}{ Clase de calidad de fuste } & \multicolumn{2}{c}{$\mathbf{N}^{\circ}$ individuos } \\
\cline { 2 - 9 } & 1 & 2 & 3 & 4 & 5 & 6 & Total & $\%$ \\
\hline Fustales & 5 & 88 & 114 & 37 & 16 & 13 & 273 & 28,4 \\
Latizales & 24 & 289 & 247 & 80 & 29 & 13 & 682 & 71,0 \\
Brinzales & 0 & 1 & 0 & 0 & 0 & 5 & 6 & 0,6 \\
Total & 29 & 378 & 361 & 117 & 45 & 31 & 961 & 100,0 \\
\hline$\%$ & 3 & 39,3 & 37,6 & 12,2 & 4,7 & 3,2 & 100,0 & 100,0 \\
\hline
\end{tabular}


el 10,41\% y 12,38\% corresponden a estas categorías. En el caso de los latizales existe un mayor número de individuos, el 33,51\% corresponden a la categoría tolerable y 17,07\% muy pobre (Tabla 2).

Los resultados muestran que, del total de la población en la categoría de latizales, el 30,07\% de individuos presentan fustes bien rectos y cilíndricos, sin defectos y 25,70\% presentan fuste recto en la mayor parte de su longitud. Para el grupo de fustales, la proporción es menor; solo $9,15 \%$ tienen fustes bien rectos y cilíndricos, sin defectos y 11,86\% con fuste recto en la mayor parte de su longitud (Tabla 3).

\section{DISCUSIÓN}

De acuerdo a la forma de la distribución del número de árboles por clases diamétricas el rodal presenta una estructura coetánea. La cual corresponde a que la mayor parte de los individuos de la cobertura vegetal tiene una misma edad o tamaño, o los individuos están concentrados en una misma clase de edad o tamaño. Esta estructura se expresa gráficamente con una curva en forma de campana, mientras que en una estructura discetánea, donde los individuos del bosque se encuentran distribuidos en varias clases de tamaños, se representa mediante una distribución del tipo J invertida (Louman et al., 2001).

La densidad del rodal es superior a lo reportado por Ugarte \& Dominguez (2010) en plantaciones de capirona de 64 meses de edad en la cuenca del río Aguaytía región Ucayali, 788,8-1032 individuos por ha; así como en campos bajo sistema de manejo de barbecho de 43 años en zonas inundables de la región Loreto con 930 árboles por ha De Jong (2001). Del mismo modo comparado con otras especies de comportamiento y hábitat similar como Guazuma crinita (Bolaina), Padoch et al., (2008), Putzel et al., (2013) mencionan la existencia de más de 1200 árboles por ha en barbechos jóvenes $(<3$ años).

La existencia de aproximadamente 425 individuos por ha con diámetros superiores a $10 \mathrm{~cm}$ es mayor a la densidad de capirona de los bosques inundables maduros de la Amazonía brasilera, donde Freitas et al., (2019) encontró 28,39 individuos por ha. A nivel de densidad total del bosque para árboles mayores de 10 $\mathrm{cm}$, nuestros resultados son similares al bosque latifoliado maduro de la llanura aluvial inundable en la Amazonía peruana (restinga alta, baja y tahuampa) que está entre 320-560 individuos por ha (Zárate et al., 2013; Honorio et al., 2015) y a los de la Amazonía brasilera con 478 (Freitas et al., 2019).

La madera aprovechada del rodal presenta dimensiones de alturas superiores a 2,5 m y diámetros mayores de $5 \mathrm{~cm}$ y por lo tanto debe tener elementos estructurales de madera redonda similares a los presentados por Espíritu \& Panduro (2010). Este tipo de madera se sugiere ser utilizado para la construcción de viviendas rurales como vigas, viguetas, largueros, caibros, cumbreras, horcones, viga de techo, crucetas e interior de armadura, y cuyas dimensiones necesarias son de 5-15 cm de diámetro y 2,5-12 $\mathrm{m}$ de longitud. De acuerdo a nuestra legislación actual, en el Perú solo se pueden extraerárboles de Calycophyllum spruceanum a partir de los $41 \mathrm{~cm} \mathrm{de}$ DAP dentro de concesiones forestales maderables (Resolución Jefatural № 458-2002-INRENA). Sin embargo, aún falta normar el aprovechamiento de la madera redonda en el país, y especialmente de bosques inundables.

El área basal es un parámetro que mide el potencial del bosque. El rodal a pesar de su corta edad estimada en ocho años, presenta aproximadamente $12 \mathrm{~m}^{2}$ por ha de área basal. Ángulo \& Fasabi (2016) reportaron un área 
basal de 18,4 a $27,5 \mathrm{~m}^{2}$ por ha en plantaciones de capirona de 10 años en Cerro Escalera (Departamento de San Martín, Amazonía peruana). Sin embargo, el área basal de nuestro rodal es superior a lo reportado para la especie por Nebel et al., (2001) en zonas inundables de la Amazonía peruana $2,78 \mathrm{~m}^{2}$ por ha. Así como en Brasil $2 \mathrm{~m}^{2}$ por ha (Freitas et al., 2019); pero inferior al potencial de estos bosques estimados en 20-29 $\mathrm{m}^{2}$ por ha (Nebel et al., 2001) y entre 16,07-38,33 $\mathrm{m}^{2}$ por ha (Carim et al., 2017) en la Amazonía brasilera.

Callophyllum spruceanum, está considerada como una especie heliófita de rápido crecimiento desde su establecimiento en el bosque donde se instala principalmente en estadíos tempranos de la vegetación (Baluarte \& Nebel, 2002), así mismo se desarrolla en terrenos fértiles con alta cantidad de nutrientes como los suelos de los bosques inundables (Lucas et al., 2012). Estudios realizados en plantaciones de Calycophyllum spruceanum con fertilización demuestran la influencia significativa de los nutrientes en el rápido crecimiento de la especie (Vilchez, 2019). Sin embargo, existen otros factores adicionales que influyen en el crecimiento de las plantas, como fisiografía, factores climatológicos (Villasana \& Suárez, 1997; Dambroth \& Bassam, 1983), y factores genéticos (Alencar, 1994., Sotelo et al., 2006; Cornelius et al., 2018).

Al parecer las bajas condiciones deluminosidad en el grupo de latizales afectan las formas de copas, de este modo existen un alto número de copas de medio círculo y menos de medio círculo. Synnot (1991) manifiesta que las clases de forma de las copas menos de medio círculo (Pobre) y solo una o pocas ramas (Muy pobre) representan árboles con pocas posibilidades de crecimiento o reacción a tratamientos silviculturales, mientras que las clases círculo completo (Perfecta) y círculo irregular (Buena) son óptimas.
Debido a que existe un alto número de individuos que reciben deficiente cantidad de luz, esta situación podría ser la causa de la existencia de gran cantidad de plantas que presentan fuste recto en la mayor parte de su longitud y parcialmente circular, pero que podrían ir empeorando la calidad del fuste en el futuro de no mejorarse las condiciones de luminosidad de las copas.

En base a los resultados se puede afirmar, con respecto a la forma de la copa como medida de la salud y vigor de un árbol, que la población del rodal conformada por los latizales se encuentra en condiciones tolerables hacia muy pobres. Mientras que para el grupo de fustales presenta un alto porcentaje de copas óptimas, al respecto Orrego \& Bustamante (2017) menciona que el porcentaje de copas óptimas va aumentando a medida que se incrementan los diámetros, ecológicamente las copas bien iluminadas poseen buena forma y crecen bien en el rodal.

Debido a que el rodal contiene un alto número de plantas dominadas principalmente por el grupo de latizales, existe por lo tanto una gran competencia entre ellas, frente a esta situación es necesario ir formando un rodal con distanciamiento óptimo, donde se procura poner a disposición del grupo de latizales la mayor cantidad de recursos, luz, agua, nutrientes y espacio; estas condiciones permitirán obtener un mayor crecimiento del árbol (Rivera et al., 2013). A pesar de que el rodal viene siendo cosechado, un conteo arrojó la cifra de 290 "tocones" (Base del árbol que queda en el bosque después de ser aprovechado), al parecer la cantidad extraída y sin planificación ha sido insuficiente como para darle las condiciones adecuadas para el crecimiento, pues actualmente existe un alto porcentaje de latizales con cantidades deficientes de luz que necesitan ser liberadas e inducir su crecimiento. Siendo necesario complementar 
las aperturas ocasionadas por la cosecha para tener una mayor y mejor distribución de árboles jóvenes que garanticen la producción de madera en el futuro.

Consideramos que el tratamiento silvicultural adecuado de acuerdo a la condición actual del rodal es un raleo de tipo selectivo-elegido con el fin de regular el distanciamiento entre los árboles mejor conformados y vigorosos y mejorar las condiciones para su desarrollo. Podría aplicarse tecnología intermedia y correctas prácticas en las operaciones de aprovechamiento forestal y transformación primaria y otros usos que se pueda dar a la madera. El producto obtenido podría estar destinado al uso en leña y en el caso de los mejores fustes como material para construcciones rurales inclusive como madera para aserrío en el caso de los fustes más desarrollados.

El rodal estudiado proviene de la implementación de un sistema gestionado por un agricultor ribereño, por lo tanto, debe valorarse el emprendimiento de esta iniciativa local. Además, no es la única iniciativa desarrollada en la zona, existen otras experiencias prácticas similares a la especie asociada con cultivos agrícolas (Cronckleton et al., 2013; Putzel et al., 2012; Correa et al., 2019). Este estudio trata de mostrar que el establecimiento de rodales de capirona a partir del manejo de la regeneración natural constituye una alternativa viabley económica para el manejo de las áreas inundables de la Amazonía. La implementación del cultivo de la capirona en predios agrícolas de áreas inundables por los ribereños amazónicos sería una alternativa que podría formar parte de la agricultura familiar, teniendo en consideración la actual promoción por el Estado peruano de acuerdo al Decreto Supremo 015 de 2016 [Ministerio de Desarrollo Agrario y Riego] que aprueba el reglamento de la la Ley de Promoción y Desarrollo de la Agricultura
Familiar. Pero para esto hace falta una asistencia técnica forestal; en la que se realicen modelos de distribución espacial de los rodales naturales, inventarios de rodales naturales, y capacitación sobre el manejo de rodales naturales. Sería importante que el mismo estado desarrolle esa asistencia técnica a través de una entidad o quizás algún Organismo no Gubernamental de Desarrollo. Esto impactaría positivamente en aquellos silvicultores artesanales amazónicos.

\section{CONCLUSIONES Y RECOMENDACIONES}

Dinámicamente el rodal se encuentra en pleno desarrollo, sin embargo, a su corta edad presenta densidades y alto rendimiento de área basal y volumen maderable superiores a plantaciones y campos de barbecho bajo manejo, lo que hace atractivo su manejo sostenible.

El grupo de latizales es el más abundante del rodal, pero que al mismo tiempo presenta un alto número de plantas con deficiente iluminación y forma de copas, por lo que la aplicación de tratamientos silviculturales debe estar orientado a favorecer este grupo de plantas, incluyendo individuos de otros grupos con buena estructura y deseadas para el aprovechamiento forestal.

El tratamiento silvicultural adecuado es un raleo tipo selectivo-elegido analizando la distribución de las plantas en rodal con el fin de dar el espaciamiento suficiente para favorecer el crecimiento del fuste en diámetro y altura y vigor de las copas. El producto obtenido del raleo podría estar destinado al uso en leña y en el caso de los mejores fustes como material para construcciones rurales inclusive como madera para aserrío.

Realizar un acompañamiento o asesoramiento al propietario del rodal con el fin de proseguir con los estudios sobre crecimiento, desarrollo 
y productividad del rodal, resultado de los tratamientos. Idealmente por una entidad estatal que brinde este tipo de asesoramiento.

Se recomienda realizar un ensayo de diferentes intensidades de raleo, que permita comparar los resultados del tratamiento y el comportamiento del rodal sin tratamiento.

\section{AGRADECIMIENTOS}

Al Señor Brígido Rengifo Terán, propietario de la finca "el cuerito" donde se encuentra ubicado el rodal natural de "capirona", por permitirnos ingresar a su predio para realizar la evaluación del rodal. A los señores Christian Pérez Mendoza y Pablo Carolus Fernández, por la ejecución de los trabajos de campo. A Rildo Rojas Tuanama, por apoyar en el procesamiento electrónico de la información de campo.

\section{REFERENCIAS BIBLIOGRÁFICAS}

Alencar, J. 1994. Fenología de cinco especies arbóreas tropicales de Sapotaceae correlacionada a variáveis climáticas na Reserva Ducke, Manaus. Manaus-Brazil. Acta Amazónica 24(3/4): 161-182. DOI: https://doi.org/10.1590/180943921994243182

Angulo, W.; Fasabi, H. 2016. Rendimiento Maderable de plantaciones Forestales establecidos en Cinco Regiones de la Amazonía Peruana. Ministerio de Agricultura y Riego - Instituto Nacional de Innovación Agraria, Pucalla. 26pp.

Baluarte, J.; Nebel, G. 2002. Incremento diamétrico de Guarea macrophylla VAHL. Y Calycophyllum spruceanum (BENTHAM) HOOKER F. EX. SCHUMANN en bosques inundables de la Amazonía Peruana. Folia Amazónica, 13(1-2): 109-120. DOI: https://doi.org/10.24841/fa. v13i1-2.140
Carim, M. J.; Wittmann, F.K.; Piedade, M.T.F.; Da Silva, J.R.; Toste, L. 2017. Composition, diversity, and structure of tidal "Várzea" and "Igapó" floodplain forests in eastern Amazonia, Brazil. Brazilian Journal of Botany, 40(1): 115-124. DOI: https://doi.org/10.1007/s40415-0160315-6

Cornelius, J.P.; Pinedo, R.; Sotelo, C.; Ugarte, J.; Weber, J. 2018. Efficiency of early selection in Calycophyllum spruceanum and Guazuma crinita, two fast-growing timber species of the Peruvian Amazon. Canadian Journal of Forest Research. 48: 1-7. DOI: https://doi.org/10.1139/ cjfr-2017-0407

Correa, V.E.; Del Castillo, D.; Hinga, H.; Paredes, E.J.; Pinedo, M.H.; Freitas, L. 2019. Agroforestería familiar inundable y procesos de adaptación al medio ambiente en Loreto. Instituto de Investigaciones de la Amazonía Peruana, Iquitos. 164pp.

Cronkleton, P.; Larson, A.M.; Pinedo-Vásquez, M.; Putzel, L.; Salazar, O.; Sears, R. 2013. Producción y comercialización de bolaina (Guazuma crinita), una especie amazónica de rápido crecimiento. Un llamado a la adopción de un marco de políticas que apoye los medios de vida. CIFOR, Bogor - Indonesia. 6pp. DOI: https://doi.org/10.17528/cifor/004257

Decreto Supremo 015 de 2016 [Ministerio de Desarrollo Agrario y Riego]. Aprueba el Reglamento de la Ley № 30355 de Promoción y Desarrollo de la Agricultura Familiar y crea la Comisión Multisectorial de Promoción y Desarrollo de la Agricultura Familiar. 22 de julio de 2016.

Dambroth, M.; Bassam, N. 1983. Low input varieties: definition, ecological requirements and selection. Plant and Soil, 72(2-3): 365-377. DOI: https://doi.org/10.1007/BF02181974

De Jong, W. 2001. Tree and forest management in the floodplains of the Peruvian Amazon. Forest Ecology and Management, 150:125- 
134. DOI: https://doi.org/10.1016/S03781127(00)00686-1

Espíritu, J.M.; Panduro, R.M. 2010. La madera redonda en la construcción de viviendas rurales en las zonas de Puerto Almendra, Zungarococha y Nina Rumi, Loreto, Perú. Conocimiento Amazónico, 1(1): 13-21.

Freitas da Luz, J., Silva dos Santos, E.; Alves, C.; de Oliveira Cruz Junior, F.; Castelo dos Santos, A. 2019. Structural analysis and distribution patterns in lowland tropical forest, eastern Amazon. Journal of Agricultural Studies, 7(4): 241-255. DOI: https://doi.org/10.5296/jas. v7i4.15366

Gorbitz, G. 2015. Estimación del carbono almacenado en la biomasa aérea de plantaciones forestales de 8 años de Calycophyllum spruceanum Benth. Xilema, 28(1): 53-60. DOI: http:// dx.doi.org/10.21704/x.v28i1.598

Honorio, N.; Vega, J.; Corrales, M. 2015. Diversidad, estructura y carbono de los bosques de los bosques aluviales del noreste peruano. $\mathrm{FO}_{\mathrm{O}}$ lia Amazónica, 24(1): 55-70. DOI: https://doi. org/10.24841/fa.v24i1

Kvist, L.P.; Nebel, G. 2001. A review of Peruvian flood plain forests: Ecosystems, inhabitants and resource use. Forest Ecology and Management, 150:3-26. DOI: https://doi. org/10.1016/S0378-1127(00)00679-4

Lucas, C.M.; Mekdece, F.; Nascimento, C.M.; Holanda, A.S.; Braga, J.; Dias, S.; Sousa, S.; Rosa, P.S.; Suemitsu, C. 2012. Effects of short-term and prolonged saturation on seed germination of Amazonian floodplain forest species. Aquatic Botany, 99: 49-55. DOI: https://doi. org/10.1016/j.aquabot.2012.02.004

Louman, B.; Quiróz, D.; Nilsson, M. 2001. Silvicultura de bosques latifoliados húmedos con énfasis en América Central. CATIE, Turrialba - Costa Rica. 265pp.

Marengo, J.A. 1998. Climatología de la Zona de Iquitos, Perú. In: Kalliola, R., Flores-Paitán, S.
(Eds). Geoecología y desarrollo Amazónico: estudio integrado en la zona de Iquitos, Perú. Annales Universitatis Turkuensis Ser A 11114. p. 35-57.

MINAM. 2015. Mapa nacional de cobertura vegetal. Memoria descriptiva. Ministerio del Ambiente, Lima - Perú. 105pp.

Nebel, G.; Kvist, L.; Vanclay, J.; Chritensen, H.; Freitas, L.; Ruiz, J. 2001. Structure and floristic composition of floodplain forests in the peruvian Amazon I. Overstorey. Forest Ecology and Management, 150(1-2): 27-57. DOI: https:// doi.org/10.1016/S0378-1127(00)00680-0

Orrego, D.R.; Bustamante, N.R. 2017. Trabajabilidad de la madera de capirona (Calycophyllum spruceanum) procedente de plantaciones de la cuenca del río Aguaytia en la región de Ucayali - Perú. Revista Forestal del Perú, 32(2): 97-106. DOI: http://dx.doi.org/10.21704/rfp. v32i2.1041

Padoch, C.; Brondizio, E.; Costa, S.; Pinedo-Vasquez, M.; Sears, R.; Siqueira, A. 2008. Urban forest and rural cities: multi-sited households, consumption patterns, and forest resources in Amazonia. Ecology and Society, 13(2): 2. DOI: https://doi.org/10.5751/ES02526-130202

Putzel, L.; Padoch, C.; Ricse, A. 2012. Putting back the trees: Smallholder silvicultural enrichment of post-logged concession forest in Peruvian Amazonia. Small-Scale Forestry, 12(3): 421436. DOI: https://doi.org/10.1007/s11842012-9221-3

Rivera, L.E.; Peñuela, M.C.; Jiménez, E.M.; Vargas, M.D.P. 2013. Ecología y silvicultura de especies útiles amazónicas: Abarco (Cariniana micrantha Ducke), Quinilla (Manilkara bidentata (A. DC.) A.Chev.) Y Violeta (Peltogyne paniculata Benth.). Universidad Nacional de Colombia (Sede Amazonia); Instituto Amazónico de Investigaciones - IMANI, Leticia - Colombia. 180pp. 
Rodriguez, F.; Bendayán, L.; Rojas, C.; Calle, C. 1991. Los suelos de la región del Amazonas según unidades fisiográficas. Folia Amazónica, 3(1-2): 7-21 DOI: https://doi.org/10.24841/ fa.v3i1-2

Sears, R.R. 2003. New forestry on the floodplain: the ecology and management of Calycophyllum spruceanum (Rubiaceae) on the Amazon landscape. Tesis de doctorado, Graduate School of Arts and Sciences, Columbia University, Ithaca. 243pp.

Synnott, T.J. 1991. Manual de procedimiento de instalación de parcela permanente para bosque húmedo tropical. Instituto Tecnológico de Costa Rica, San José. 103pp.

Sotelo, C.; Hernández, R.; Beaulieu, J.; Weber, J.C. 2006. Genetic variation and correlations between growth and wood density of Calycophyllum spruceanum Benth. at an early age in the peruvian Amazon. Silvae Genetica, 55(4): 217-228. DOI: https://doi.org/10.1515/sg2006-0029

Tello, D.; Paredes, M.; Cobos, M. 2013. Propuesta de clasificación microclimática en función a la vegetación predominante en la cuenca del río Itaya, Loreto, Perú. ECIPerú, 9(2): 84-88.

Ugarte, L.J.; Domínguez, G. 2010. Índice de sitio (IS) de Calycophyllum spruceanum Benth. en relación con la altura dominante del rodal en ensayos de plantación en la Cuenca del Aguaytía, Ucayali, Perú. Ecología Aplicada, 9(1-2): 101-111. DOI: http://dx.doi.org/10.21704/ rea.v9i1-2.400

Vilchez, N. 2019. Efecto de la fertilización en el crecimiento de Calycophyllum spruceanum (Benth) Hook F. en plantación, en el Centro de Investigación y Producción Tulumayo - Leoncio Prado. Tesis de pre-grado, Universidad Nacional del Centro del Perú, Facultad Ciencias Forestales y del Ambiente, Huancayo, Perú. $71 \mathrm{pp}$.

Villasana, R.; Suárez, A. 1997. Estudio fenológico de dieciséis especies forestales presentes en la Reserva Forestal Imataca, Estado Bolívar -Venezuela. Revista Forestal Venezolana, 41(1): 1321.

Weber, J.C.; Sotelo, C.; Vidaurre, H.; Dawson, I.; Simons, T. 1999. Out of the woods-domesticating capirona. Agroforestry Today, 11(3-4): 4-6.

Zarate R.; Mori, T.J.; Maco, J.T. 2013. Estructura y composición florística de comunidades vegetales del área adyacente de la carretera Iquitos - Nauta, Loreto - Perú. Folía Amazónica, 22(12): 77-89. DOI: https://doi.org/10.24841/ fa.v22i1-2

Recibido: 14 de marzo de 2021 Aceptado para publicación: 2 de junio de 2021 